\title{
Discussion of Standard Specimens for Performance Evaluation in Atom Probe Tomography
}

\author{
B. P. Gorman* and T. F. Kelly**
}

* Department of Metallurgical and Materials Engineering, Colorado School of Mines, Golden, CO 80401

** Cameca Instruments, Inc., 5500 Nobel Dr., Madison, WI, 53711

Materials for use as standard specimens in atom probe tomography (APT) must have several unique aspects that are not typically considered in other spectroscopy techniques. This is due to the inseparable interpretation of compositional and spatial information contained in APT data analysis and subsequent reconstruction. A wide range of specimen preparation, instrumental acquisition, and reconstruction standards are required for accurate definitions of instrumental performance. Unique data acquisition and reconstruction algorithms require that the community adopt similarly unique definitions specific to compositional (or mass) resolution and spatial resolution.

In any measurement of instrument performance, the input function should ideally be as sharp as possible (delta function) to maximize the measurement's sensitivity to any effects of the instrument on the output. In APT, the specimen is the primary input function. Thus the specimen properties should be as ideal as possible relative to the performance that is to be measured. Measurements of spatial resolution and mass resolution in APT are strongly influenced by the specimen material properties (thermal diffusivity, anomalous absorption depth, melting temperature, surface diffusivity, ...) and geometry (tip radius, shank angle, crystal orientation, ...). Furthermore, the measurements need to be repeatable both within a laboratory and among multiple laboratories. The ideal standard specimen for spatial and mass resolution tests therefore should have optimized values for properties that influence spatial and mass resolution and it should have reproducible specimen geometry.

Silicon pre-sharpened microtip arrays, Fig. 1, are very close to meeting all of these criteria. Silicon has very good thermal diffusivity at cryogenic temperatures. It has a relatively high melting temperature which reduces thermal migration. The anomalous absorption depth is low especially for ultraviolet wavelengths. It is available in reproducible compositions of very high purity. It is defect free. The orientation is always $<100>$ and the microtip geometry is highly regular. Note that the microtip geometry will work well for local electrode geometries but cannot be used in remote electrode geometries. It is possible to liftout a microtip and attach it to a metal needle but the reproducibility of this geometry would need to be investigated. Fortunately, with the advent of insitu liftout FIB specimen preparation techniques [1], standardized protocols can be implemented across the community.

In addition to specimen preparation, standardized techniques for monitoring the laser fluence on APT specimens are needed. Specifically, methods for quantifying the photon flux out of the laser optics, and for determining the fraction of the focused laser spot that is incident on the specimen are needed. Alternatively, standardized methods for in-situ monitoring of the specimen temperature during pulsing would be equally valuable as this method would also take into account the amount of laser light lost to reflection or diffuse scattering.

Before reconstruction of APT data, time-of-flight spectra must be interpreted such that certain TOF bins are assigned ("ranged") to a certain element or cluster of elements. Each bin in a TOF spectrum typically corresponds to $0.001 \mathrm{Da}$, where atomic masses range from 1 to $>500 \mathrm{Da}$. Defining which 
bins constitute the onset of a particular ion species is thus up to user interpretation (Fig. 2). Currently, no standards exist for the definition of mass ranges, primarily due to the wide variance in mass resolution observed between both hardware and specimen arrangements. It should be noted that a small change in specimen position or hit position on the microchannel plate can define the mass resolution of the system. Standard protocols for specimen positioning are thus encouraged.

Once position-sensitive TOF data are acquired via standardized protocols, reconstruction algorithms must be implemented for data interpretation. While evaporation fields for most atoms are theoretically calculated at absolute zero, the chemical nature of the specimen does not always yield correct reconstructions based on those fields. Additionally, the temperature dependence of the evaporation field varies widely among the elements, and has not been well quantified. A detailed study of evaporation field, calculated via specimen geometries for a wide variety of materials, is highly suggested. An additional understanding of field evaporation and trajectory aberrations in dielectrics is also required, but virtually nothing is known about field and heat distributions during these experiments. This enormous undertaking is suggested to be a highly coordinated effort from everyone who utilizes APT.

Based on the prior discussion, idealized specimens for atom probe tomography are suggested. For instance, a single element, amorphous material (such as a-Si) would allow for the quantification of system hardware in terms of TOF standardization without the added issues of preferential evaporation from crystal facets.

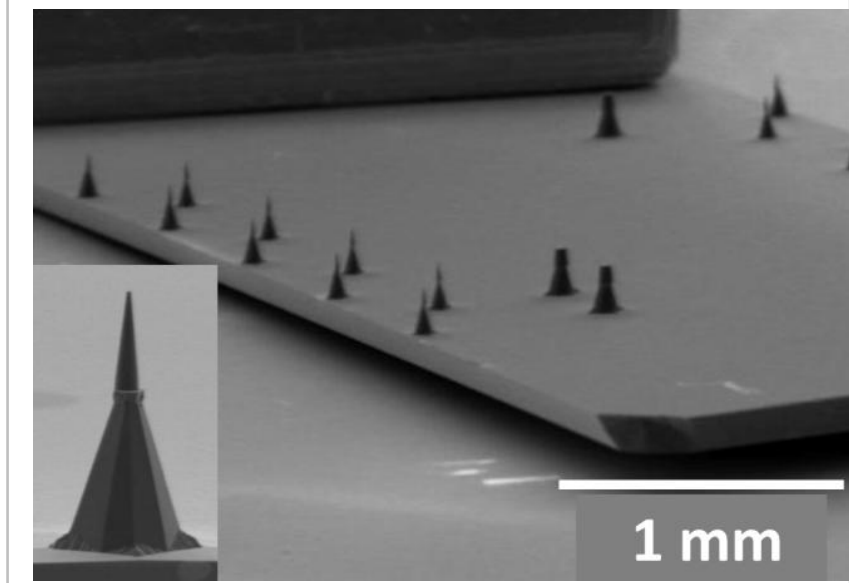

FIG. 1. Pre-sharpened microtip array made of $<100>$ Si wafer. The microtips are about 100 micron tall with $<100>$ normal to the wafer surface.

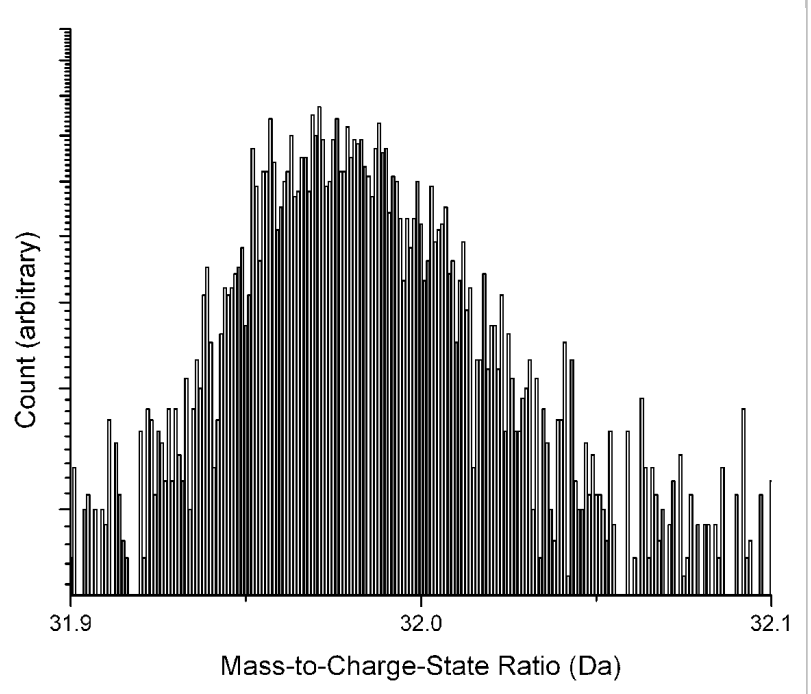

FIG. 2. Representative mass spectrum of $\mathrm{O}_{2}^{+}$ illustrating the distribution of bins with respect to the ion mass resolution.

\section{Reference}

[1] K. Thompson, D. Lawrence, D. J. Larson, J. D. Olson, T. F. Kelly, and B. Gorman, Ultramicroscopy 107 (2007) 131-139. 